\title{
Nonlinear plasmonic metasurfaces assisted laser mode locking
}

\author{
Lei Zhang, ${ }^{1,2}$ Jiyong Wang, ${ }^{1,2^{*}}$ Aurelien Coillet, ${ }^{3}$ Philippe Grelu, ${ }^{3}$ \\ Benoit Cluzel, ${ }^{3 *}$ Min Qiu ${ }^{1,2 *}$ \\ ${ }^{1}$ Key Laboratory of 3D Micro/Nano Fabrication and Characterization of Zhejiang Province, School of Engineering, Westlake University, 18 \\ Shilongshan Road, 310024 Hangzhou, Zhejiang Province, China \\ ${ }^{2}$ Institute of Advanced Technology, Westlake Institute for Advanced Study, 18 Shilongshan Road, 310024 Hangzhou, Zhejiang Province, \\ China \\ ${ }^{3}$ Laboratoire Interdisciplinaire Carnot de Bourgogne, Université Bourgogne Franche-Comté, 9 avenue Alain Savary, 21078 Dijon, France
}

\begin{abstract}
Plasmonic metasurfaces are artificial 2D layers made of subwavelength elementary cells, which give rise to novel wave properties that do not exist in nature. In the linear regime, their applications have been extensively studied, especially in wavefront manipulation for lensing, holography or polarization control. Interests in metasurfaces operating in nonlinear regime have also increased due to their ability to efficiently convert the fundamental light into harmonic frequencies and multiphoton emissions. Nevertheless, practical applications in the nonlinear regime have been rarely reported.

In this study, we report that plasmonic metasurfaces with well-controlled polarimetric nonlinear transfer functions perform as saturable absorbers with modulation performances superior to that of other 2D materials. We employ planar nanotechnologies to fabricate 2D plasmonic metasurfaces with precise size, gap and orientation. We quantify the relationship between saturable absorption and the plasmonic resonances of the unit cell by altering the excitation power of pumping laser, the polarization of incident light and the geometrical parameters of the plasmonic metasurfaces. Finally, we provide a practical implementation by integrating the saturable metasurfaces into a fiber laser cavity and realize a stable self-starting ultrashort laser pulse generation. As such, this work sheds light on ultrathin nonlinear saturable absorbers for applications where nonlinear functions are required, such as in ultrafast laser or neuromorphic circuits.
\end{abstract}

\section{References:}

[1] Wang, J., Coillet, A., Demichel, O. et al. “Saturable plasmonic metasurfaces for laser mode locking,” Light Sci Appl 9, 50 (2020).

[2] Sain, B., Zentgraf, T. “Metasurfaces help lasers to mode-lock,” Light Sci Appl 9, 67 (2020). 